\title{
Mayores, publicidad y medios de comunicación: Una revisión teórica
}

\author{
José F. Mancebo-AraciL \\ Universidad de Alicante \\ josef.mancebo@gmail.com
}

\begin{abstract}
Resumen:
El presente trabajo tiene como objetivo revisar los principales estudios que relacionan a las personas mayores con los medios de comunicación o publicidad, y cómo son representados. Muchos de estos estudios revelan que la imagen de los mayores ha sido tradicionalmente infrarrepresentada, infravalorada y estereotipada, tanto en programas como anuncios. Las industrias del entretenimiento y publicitaria deben adquirir mayor conciencia sobre su (falta de) estrategia de segmentación de los mayores de 55 años, un nicho con cada vez mayor peso específico y más relevante en los países industrializados.
\end{abstract}

Palabras clave: Mayores; Publicidad; Representación; Medios de Comunicación; Televisión.

\section{Elderly, advertising and media: A theoretical review.}

\begin{abstract}
:
This paper aims to review the main studies relating to elderly people with media and advertising, and how they are represented. Many of these studies show that the image of the elderly has been traditionally underrepresented, undervalued and stereotyped both programs and ads. The entertainment and advertising industries must become more aware about their (lack of) segmentation strategy over 55 years a growing niche with specific weight and more relevant in industrialized countries.
\end{abstract}

\section{Referencia normalizada:}

Mancebo-Aracil, J. F. (2014): Mayores, publicidad y medios de comunicación: una revisión teórica. Historia y Comunicación Social. Vol. 19. Núm. Especial Febrero. Págs. 573-588.

Sumario: 1. Introducción. 2. Metodología. 3. Resultados. 3.1. Publicidad y Mayores. 3.2 Medios de comunicación y mayores. 3.2.1. Televisión y prime-time. 3.2.2. El mayor entre varios medios. 3.2.3. 3.3. Otros estudios recientes. 4. Conclusiones. 4.1. Infrarrepresentados e infravalorados. 4.2. Otro grupo poblacional vulnerable. 4.3. Mejor tratados en anuncios que en series. 4.4. Retos futuros en el estudio del mayor, medios y publicidad. 5. Bibliografía.

\section{Introducción}

Las personas mayores conforman, en sí mismo, todo un segmento de mercado, homogéneo en muchos sentidos pero segmentable incluso por subgrupos de edad. Sus miembros se comportan como consumidores cada vez más exigentes y conocedores de los medios de comunicación y de la publicidad (Hudson, 2009). 
Esta realidad se contrapone con el hecho de que el mayor no ha sido (ni de hecho, es) tratado, ni representado, sea en series de ficción, programas o publicidad, de acuerdo a la realidad social que describimos. El presente trabajo pretende realizar un recorrido teórico por la investigación académica que relaciona mayores, medios y publicidad, con el objeto de establecer conclusiones encaminadas a la reflexión general, y de la industria publicitaria o la televisiva, en particular, especialmente de los países más desarrollados, con mayor esperanza de vida y, por tanto, más envejecidos.

\section{Metodología}

Como veremos posteriormente, para este estudio, se han manejado más de 70 referencias, tomadas de revistas españolas y anglosajonas indexadas en las principales bases de datos, Scopus, ISI-Wok, ESBCO y, en el caso español, In-Recs y Latindex. Para la búsqueda y selección de los artículos se utilizaron las siguientes palabras clave (Tabla 1):

Tabla 1. Palabras clave en la búsqueda de artículos científicos indexados.

\begin{tabular}{|l|l|}
\hline Campo semántico & Palabras Clave (Keywords) \\
\hline \multirow{2}{*}{ PERSONAS MAYORES } & Sénior, Mayores, Tercera Edad, Envejecimiento, Representación \\
\cline { 2 - 2 } & Elderly, Aging, Older People, Senior, Boomers, Portrayal \\
\hline \multirow{2}{*}{ PUBLICIDAD } & Publicidad, Anuncios, \\
\cline { 2 - 2 } & Advertising, Adverts \\
\hline \multirow{2}{*}{ COMUNICACIÓN } & $\begin{array}{l}\text { Television, Medios de Comunicación } \\
\text { Television, Media }\end{array}$ \\
\hline
\end{tabular}

Elaboración propia.

Los estudios encontrados fueron clasificados en tres áreas temáticas:

- Publicidad

- Contenido no publicitario: Series y ficción televisiva, programas, noticiarios, etc.

- Otros estudios: Búsqueda de la opinión del mayor, etc.

\section{Resultados}

Los primeros resultados extraídos de estas búsquedas muestran que existen más referencias sobre mayores y publicidad que cualquier otro tema que relacione mayores y medios de comunicación. (Gráfico 1): 
Gráfico 1. Estudios analizados sobre representación del mayor en medios (1974-2013).

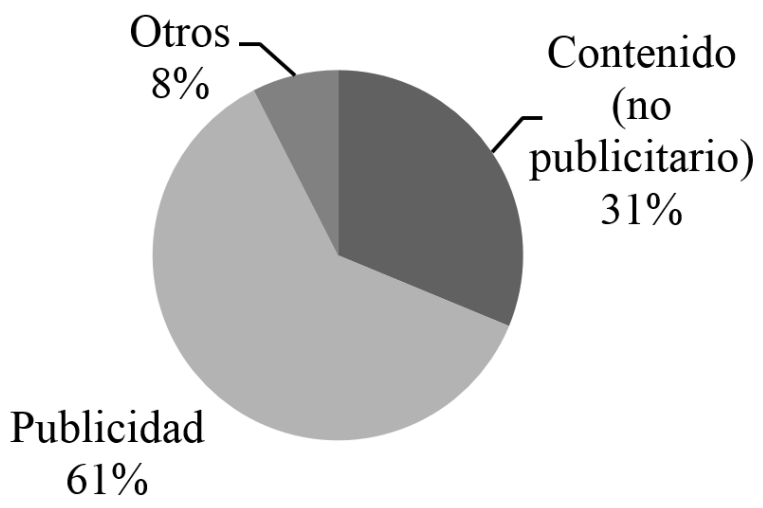

Elaboración propia.

En concreto, los estudios analizados, por temática principal, han sido los relacionados en Tabla 2.

Tabla 2. Relación de trabajos analizados de mayores y medios, por temática de estudio.

\begin{tabular}{|l|l|}
\hline Ámbito & \multicolumn{1}{|c|}{ Estudios } \\
\hline \multirow{5}{*}{ Publicidad } & Atkins, Jenkins, \& Perkins (1991); Balazs (1995); Bing Zhang y Harwood (2004); Bing \\
& Zhang, et al. (2006); Carrigan y Szmigin (1999-B); Carrigan y Szmigin (1998); Carrigan \\
& y Szmigin (1999-A); Carrigan y Szmigin (200); Carrigan y Szmigin (2003); Cheng \\
& (1998); Cheng y Schweitzer (1996); Davis y French (1989); Dubois (1997); Festervand y \\
& Lumpkin (1984); Freixas (1998); Gantz, Gatenberg y Rainbow (1980); Greco (1988-A); \\
& Greco (1988-B); Hiemstra et. al (1983); Kvasnicka, Beymer y Perloff (1982); Lee, Kim y \\
& Han (2006); McConatha, Schnell y McKenna (1999); Miller, Leyell y Mazachek (2004); \\
& Peterson (1992); Peterson (1995); Pochintest (2012); Prieler et al. (2008); Prieler et al. \\
& (2011); Raman et al. (2008); Ramos-Soler y Carretón-Ballester (2012); Ramos-Soler y \\
& Mancebo-Aracil (2013); Roberts y Zhou (1997); Robinson y Umphrey (2006); Robinson, \\
& Gustafson y Popovich (2008); Robinson, Popovich, Gustafson y Fraser (2003); Roy y \\
& Harwood (1997); Schewe (2001); Simcock y Sudbury (2006); Smith (1976); Smith, \\
& Moschis y Moore (1987-A); Smith, Moschis y Moore (1987-B); Stephens y Warrens \\
& (1983); Swayne y Greco (1987); Szmigin y Carrigan (2001); Tupper (1995); Ursic, Ursic \\
& y Ursic (2006); Valls-Fernández y Martínez-Vicente (2007); Westerhof et al. (2010); \\
Zhou y Chen (1992)
\end{tabular}

Elaboración propia. 
La tendencia de producción de este tipo de estudios es creciente, existiendo cada vez más referencias que relacionan mayores y su representación en medios de comunicación, mayores y publicidad, u otros estudios relacionados con los mayores y medios (Gráfico 2).

Gráfico 2. Evolución $\mathrm{n}^{\mathrm{o}}$ investigaciones mayores, medios y publicidad (por década).

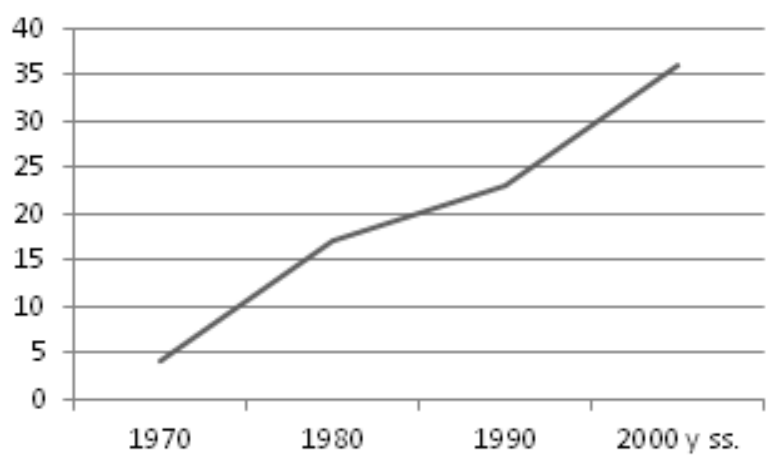

Elaboración propia.

\subsection{Publicidad y Mayores}

En el estudio pionero, Smith (1976) realizaba un análisis de contenido sobre anuncios en dos revistas especializadas. En cuanto a la elección de medios, el estudio de los mayores y publicidad se ha centrado, mayoritariamente, en revistas especializadas o medios impresos, como es el caso de los primeros estudios (Gantz et al., 1977; Gantz et al., 1980; Kvasnicka et al, 1982). Hasta 1983 no se conocerá el primer trabajo sobre publicidad televisiva y mayores.

Hiemstra et al. (1983) se puede considerar primer estudio sobre mayores en spots publicitarios. En la misma década de los ochenta existen más en este sentido, centrados en televisión y con el objeto de estudiar la representación de modelos mayores en anuncios publicitarios (Stephens et Al, 1983; Swayne et Al., 1987). Posteriormente, el mayor será tratado como consumidor complejo y consultor de fuentes de información para establecer su decisión de compra (Davis y French, 1989; Festervand et Al, 1984; Smith et Al., 1987-A; Smith et Al, 1987-B).

Algunos autores como Greco (1988-A y 1988-B) buscarán, además, la opinión de profesionales de la comunicación respecto al conocimiento y uso de modelos mayores en publicidad.

En la misma época, en España, sólo se documenta un pequeño documento, no un estudio propiamente dicho: Aparicio, 1988. Este ensayo breve critica el excesivo paternalismo y falsedad estereotipada con que los medios de comunicación de masas tratan a las personas mayores. 
A partir de 1990, se continúa en cierto sentido con la actividad desarrollada una década antes, con mayor protagonismo de la televisión a medida que pasan los años, sin existir, hasta hoy, consenso sobre la edad de consideración de la persona mayor.

Más allá de la literatura anglosajona, se documentan también estudios en idiomas como el francés (Dubois, 1997) o español (Freixas, 1998), éste último primer referente en nuestro país.

En los últimos años de la década de los 90 , se encuentran las dos autoras más prolíficas de este periodo. De 1998 a 2003, Carrigan y Szmigin publicaron seis trabajos que denotan el carácter moderno de este tipo de estudios: 1/ El mayor se considera a partir de edades jóvenes, incluso a partir de 50 años (Carrigan y Szmigin, 1998); 2/ Los artículos van acompañados de profundas revisiones teóricas e incluso principalmente teoréticos (Carrigan y Szmigin, 1999-A y 1999-B; Carrigan y Szmigin, 2000; Szmigin y Carrigan, 2001); 3/ Se retoma el interés por trabajar con la industria publicitaria (Carrigan y Szmigin, 2003); 4/ Mayor rigor metodológico.

Tras la obra de las autoras británicas, destaca, la producción de estudios a cargo de Robinson et al., con cuatro trabajos reseñables $(2003,2006,2007,2008)$, en los que se utilizará recurrentemente la "Metodología Q", cuestionarios ranking basados en las metodología de Stephenson (1953). Son años de buenos artículos teóricos (Robinson et al., 2006) y estudios completos (hermanos Ursic, 2006), que bien apuntan a un país concreto (Prieler, et al., 2008; Simcock y Sudbury, 2006; Valls-Fernández et al., 2007) o con perspectiva trasnacional (Bing Zhang et al., 2006; Lee et al., 2006). Por último, otros trabajos mantendrán un marcado carácter ensayístico y crítico (Mulley; 2007; Hudson, 2009).

\subsection{Mayores y medios de comunicación}

La investigación académica se ha centrado, sobre todo, en televisión, según la búsqueda realizada en las distintas bases de datos. No se detecta, como en el caso de la publicidad, estudios del mayor representado en otros medios de comunicación (radio o cine, por ejemplo), salvo alguna referencia aislada sobre representación de mayores en literatura (el caso de Berman y Nelson, 1987). Como se apuntaba anteriormente, los trabajos sobre mayores en los medios están principalmente centrados en el estudio la representación de los mayores en ficción televisiva, y en concreto, series o programas en prime-time, fundamentalmente en Estados Unidos. Tal y como apunta Signorelli (2004), a partir de la proliferación de la TV por cable, la mayoría de estudios se centrarán en estos soportes, dado que 'la proliferación de estos canales hacen posible que muchos telespectadores sigan de forma constante las series, llegando a alcanzar hasta la mitad de la población (Nielsen, 2000).

\subsubsection{Televisión y prime-time}

En los años 70, encontramos las dos monografías pioneras y, en cierto aspecto, claros antecedentes de muchos estudios posteriores. Aronoff (1974) realizó el primer análisis sociodemográfico que relaciona los personajes aparecidos en ficción televi- 
siva en prime-time y la residual presencia los mayores. En el mismo sentido trabajaría Northcott (1975), afirmando la preferencia por mostrar personajes de edad media en TV y, por tanto, relacionando infancia y vejez como segmentos de débil representación en los medios.

En la década de 1980 se alcanzará la madurez en este tipo de estudios. Son los casos de las primeras tesis universitarias referenciadas (González, 1979) o nuevos análisis de contenido de la presencia de mayores en series diarias (Cassata et al., 1980; Elliot, 1984), todos ellos centrados en EEUU.

Especial mención merece Gerbner et al (1980), trabajo en el que se realiza el primero y uno de los más completos análisis de contenido, de carácter diacrónico, sobre mayores y televisión. Se estudiaron personajes y programas en el periodo 19691978 y se relacionaron con los roles y la realidad de los mismos colectivos de edad de la sociedad civil norteamericana.

En Bell (1992) se analiza las imágenes de edad representada en cinco series de máxima audiencia en 1989, en la TV estadounidense. El mayor en prime-time será objeto de distintos estudios en años siguientes, como Spring (1993), Signorelli y Bacue (1999), Signorelli, (2004) Taylor (1995).

En Signorelli (2004) se analiza el contenido de 14 series en el período 1993-2002, de nuevo en prime-time, EEUU y TV por cable. Se concluyó que la proporción hombre/mujer y la proporción de blancos y minorías u hombres y mujeres no se refleja respecto a la población real. Además, los personajes femeninos son más jóvenes que los personajes masculinos

Esta última idea, conocida como 'doble estándar sexista' (infrarrepresentación de la mujer junto con la preferencia por proyectar a mujeres jóvenes) coincide con la línea ya planteada por otros autores, como Vasil, \& Wass (1993). El concepto fue acuñado por England et Al. (1981).

No es de extrañar que la mayoría de estudios se centren en series o programas con horario de máxima audiencia ya que, aunque las muestras son de conveniencia, parece más conveniente analizar el programa (o un soporte, como vimos en la publicidad), con el máximo número de seguidores. Estudiando la representación poblacional del prime-time (periodo de máxima audiencia y, por tanto, más exitoso), conseguimos no sólo obtener muestras más representativas sino conocer si el mayor está presente en los momentos estrella de la televisión y, por tanto, de mayor calado social y cultural.

Nos detendremos, por su perspectiva global más allá de la edad, en Harwood y Anderson (2002). Estos autores norteamericanos comparan la representación de la población en ficción televisiva (en EEUU, cable, series dramáticas y comedias en prime-time) con la población real del país, respecto a tres variables sociodemográficas básicas: Sexo, Edad, Raza (Grupos étnicos).

Durante la revisión teórica, los autores repasan como mientras otros investigadores no vieron falta de representación de los mayores (Dail, 1988; Peterson, 1973), muchos 
otros autores sí apreciaron que los mayores no se encontraban representados, ni en cantidad, ni respecto a valores positivos (Aronoff, 1974; Gerbner et al., 1980; Greenberg et al., 1979; Northcot, 1975; Signorelli et Gerbner, 1978). El estudio concluye, de forma crítica, varios aspectos contemplados que relacionan no sólo la poca presencia del segmento mayor en prime-time, sino que ocurre algo similar con otros grupos poblacionales, como la infancia, mujeres o minorías étnicas: 1/ Los mayores, niños y mujeres están infrarrepresentados en sitcoms y dramas en EEUU en prime-time; 2/ Por el contrario, los blancos, hombres y de edad media están sobrerrepresentados en el mismo contexto. 3/ Las mujeres tienden a estar sobre representadas en edad joven y al contrario en edad mayor (doble sexismo). 4/ Los mayores tienden a mostrar valores más negativos que la juventud. 5/ Los personajes latinos, infrarrepresentados.

Otro estudio reciente, Ye y Ward, (2010) estudia qué tipo de dolencias y enfermedades son representadas en dos populares series de TV norteamericanas: ER (en España, Urgencias) y Grey's Anatomy. En este nuevo análisis de contenido se concluyó que de los pacientes representados un 6,9\% eran entre 55-64 años; y sólo un $5,1 \%$ mayores de 65 .

Gráfico 3. Edad 'pacientes' en Anatomía de Grey y Urgencias.

A partir de Ye y Ward, (2010).

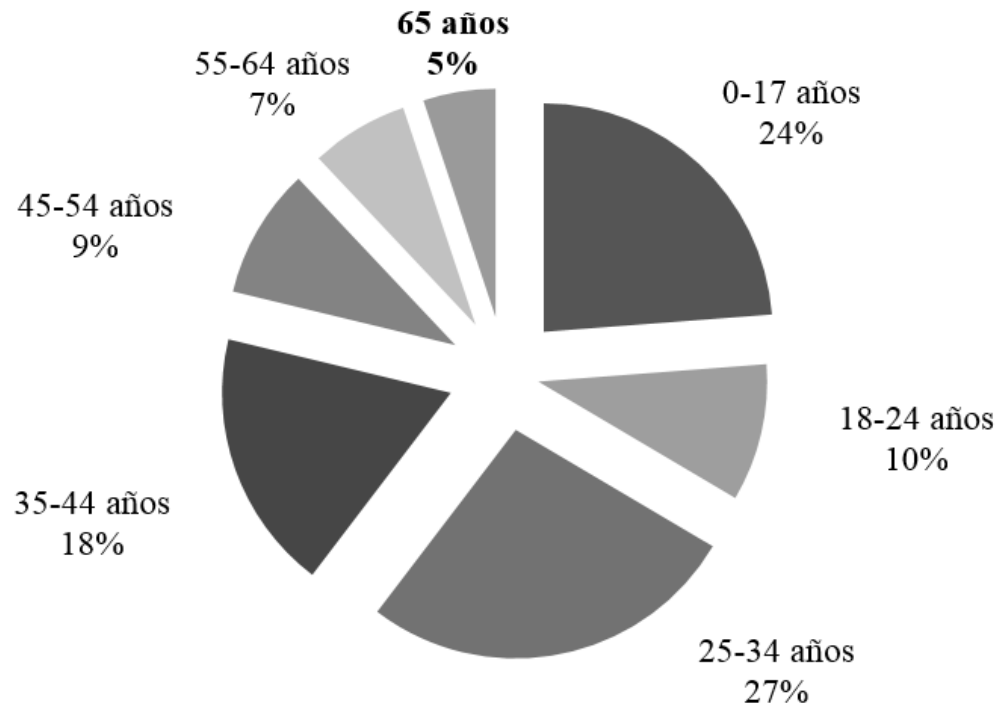

Elaboración propia.

El estudio de los mayores representados en ficción televisiva de temática de salud y los medicamentos, pese a su gran recorrido, no ha sido estudiado todo lo que cabría esperar, salvo en los casos mencionados u otros casos aislados (Hodgetts et al. 2003; Signorelli, 1983). 
Robinson, Skill y Turner, J. (2004) resulta uno de los más completos trabajos de mayores y medios de comunicación, en el que, además se pone en relación tanto patrones y representación de los mayores en distintos medios como sus consecuencias respecto al consumo en general, relaciones con otras personas, percepción social, etc. Tomando datos de consumo de medios, programas, etc. de otros autores (principalmente, Mundorf y Brownell, 1990), los autores relacionan la preferencia y el ranking de distintos medios y programas según distintos núcleos de edad Nielsel (2000), relacionado con los personajes que salen representados. Además de la televisión, analizan medios como periódicos, revistas, radio y ordenadores (con y sin Internet). Posteriormente se relaciona, en una revisión bibliográfica, la imagen de los mayores en los medios. Los mayores invierten más del $40 \%$ de su tiempo de su tiempo de ocio en consumir televisión, ver películas, escuchar música o la radio (Spring, 1993). Sin embargo, no existen estudios que relacionen mayores y publicidad, en medios como la radio (Ramos-Soler y Mancebo Aracil, 2013).

La mayoría de los estudios los mayores de 65 años no aparecen representados ni en el $5 \%$ del total de los personajes, aunque signifiquen mucho más en la población real o la audiencia que está viendo esos mismos programas (Tabla 3 ).

Tabla 3. Representación mayores de 65 años en ficción televisiva en prime-time. A partir de Robinson, et al. (2004)

\begin{tabular}{|c|c|c|c|}
\hline Estudios* & $\begin{array}{c}\text { Representación del } \\
\text { mayor }\end{array}$ & $\begin{array}{c}\text { Tot } \\
\text { al }\end{array}$ & $\%$ \\
\hline $\begin{array}{c}\text { Arnoff, 1974; Bell, 1992; Elliot, 1984; Gerbner et al., } \\
\text { 1980; Greenberg et al., 1980; Northcott, 1975; } \\
\text { Robinson \& Skill, 1995; Signorelli \& Gerbner, 1978 }\end{array}$ & $-5 \%$ & 8 & $72,73 \%$ \\
\hline Cassata et al., 1980; Elliot, 1984. & Entre el 5-10\% & 2 & $18,18 \%$ \\
\hline Peterson (1993) & Más del 10\% & 1 & $9,09 \%$ \\
\hline \multicolumn{2}{|c|}{ TOTAL } & $\mathbf{1 1}$ & $\mathbf{1 0 0 , 0 0 \%}$ \\
\hline
\end{tabular}

\subsubsection{El mayor entre varios medios}

A partir de 1990, ciertos estudios tratan de analizar las preferencias y gustos del mayor, mientras que otros, analizan el papel y la representación de los mayores en varios medios simultáneamente, o bien en otros formatos y programas distintos a las series televisivas.

Goodman (1990) investiga las preferencias de la tercera edad en cuanto a la programación televisiva, fuentes de información y noticias. Explora en las satisfacciones de esta audiencia a la hora de conocer lo que ocurre a través del medio, mediante entrevistas personales y cuestionarios por correo a cerca de las preferencias de ver televisión para noticias nacionales. 
En el mismo sentido de recabar la opinión de los mayores, Healey, \& Ross (2002) presentan un estudio de espectadores mayores opinando sobre la TV y cómo ésta proyecta la imagen del colectivo. Entre los principales resultados expuestos, se confirma el interés de los mayores por opinar sobre un medio que conocen perfectamente y que consumen de forma muy significativa: La televisión.

Más allá de este medio, Shinar (1982) estudiaría la representación de los mayores en cuatro periódicos israelíes y su relación con la permanencia de estereotipos que son capaces de mantener los medios de comunicación. Como aspecto interesante, el autor no detecta en principio infrarrepresentación del mayor, aunque destaca que la representación de los mayores no es demasiado positiva desde el punto de vista cualitativo.

En cuanto a más esfuerzos por estudiar a los mayores en varios medios de comunicación simultáneamente, Vasil, \& Wass (1993) realizan un estudio teórico sobre la representación de la tercera edad en medios electrónicos e impresos. Si bien parece un buen precedente para seguir trabajando con muestras más complejas, resulta algo ineficiente la separación entre medios 'electrónicos' e impresos, dado que si bien pudo tener alguna utilidad metodológica para el autor, no se esgrimen diferencias en los resultados o en las conclusiones atendiendo al tipo de soporte en cuestión.

\subsection{Otros estudios recientes}

En España, en la literatura reciente revisada, merece especial mención del Congreso de Comunicación e persoas maiores, cuyas actas recogidas en Kristensen, et al. (2007) contiene comunicaciones relacionadas con comunicación, socialización o metodología en el estudio de mayores (Ballester, J., et al., 2007; Belando, 2007; Frávega, \& Carnino, 2007; Garcillán, 2007; Hernández, 2007; Polo, 2007; SirimiriVVAA, 2007).

Uno de los trabajos más importantes de los últimos años, lo encontramos en Fernández-Cid, M., Martín, A., \& Cáceres M.D. (2008) en el cual se estudia con gran detalle y extensión la imagen de las personas mayores en los medios de comunicación de masas y la opinión pública.

Más recientemente, se han realizado otros esfuerzos por estudiar la comunicación con mayores, analizar el uso y tecnología de webs dirigidas a mayores y describir cómo se usan herramientas de microblogging y redes sociales. (Carretón y Ramos-Soler, 2010). 


\section{Conclusiones}

\subsection{Infrarrepresentados e infravalorados}

Primeramente, los estudios analizados evidencian que la industria mediática y publicitaria debe tomar conciencia de que se está infrarrepresentando y devaluando la imagen social del mayor. Aunque la ficción requiere libertad creativa, idea fórmulas de invención o exageración y no tiene por qué ser fidedigna a la realidad, perdurar la representación de una sociedad excesivamente joven tiene sus consecuencias, tanto para el aprendizaje social del rol del mayor, como para la propia industria que, quizá, esté perdiendo una grandísima oportunidad, especialmente con los denominados boomers (Taylor, 1995; Hudson, 2009). Diferenciando países o culturas, resulta obvio que, con los datos de esperanza de vida o índices de natalidad, trascurridos más de 50 años desde que se produce un boom de nacimientos, el público sénior parece tomar el protagonismo, sino de los medios y de la publicidad, sí de la realidad sociodemográfica y la pirámide poblacional existente.

\subsection{Otro grupo poblacional vulnerable}

No en pocas ocasiones, se aprecian similitudes en cuanto a la posición de mayores con otros grupos poblacionales vulnerables, es decir subrrepresentados y especialmente sensibles desde el punto de vista social, tales como la infancia, mujeres o minorías étnicas. Estudios como Schmitt et al (2003) y su diseño de la muestra microsegmentado de la infancia, o revisiones teóricas como Bringué y De los Ángeles (2000) sobre publicidad, televisión y niños, son excelentes ejemplos para aplicar al estudio de los mayores. En el mismo sentido, la investigación académica de los mayores en los medios puede aprender mucho de la perspectiva centrada en minorías o diferencias de género (England et al., 1981), y viceversa.

\subsection{Mejor tratados en anuncios que en series}

Respecto a las diferencias entre publicidad y medios, el trato 'más amable' del mayor en anuncios respecto series u otros programas en los que se representan, podría tener su explicación en la misma naturaleza de la publicidad. Siguiendo a Rodríguez y Mora (2002), podemos determinar tres características fundamentales de la publicidad que explicarían este fenómeno:

1. "La publicidad se halla en una relación cómplice e intertextual con la ideología dominante cuyos valores se transmiten a través de sus formas de representación". Los anuncios, por tanto, están en cierta medida 'obligados' a gustar, especialmente a quiénes van dirigidos, por lo que su discurso suele ser positivo para gustar lo máximo (u ofender lo menos posible).

2. En la publicidad se relaciona con los objetos, no como "entes materiales mudos y ensimismados a los que se refiere en sus textos, sino como mercancías y como signos cargados de significado". Más allá de sus modelos, los productos 
y servicios en general, y los dirigidos a los mayores en particular, (medicamentos, viajes, productos del hogar) se rodean de valores y propiedades amables y positivas cuando son publicitados;

3. "La publicidad genera una relación activa con el receptor, en tanto que productor de significados implícitos en sus mensajes". Los anuncios son más cuidadosos con la imagen del mayor que, por ejemplo, una serie, debido quizá al vínculo comercial y continuado que debe favorecer. La imagen del mayor, además, es más positiva en publicaciones especializadas para mayores que en prensa general o televisión (Ramos-Soler y Mancebo-Aracil, 2013).

5.4 Retos futuros en el estudio del mayor, medios y publicidad.

Por último, destacar que, aunque la mayoría de estudios de mayores y medios se han centrado en publicidad y series televisivas, será interesante comprobar si se a publicar sobre otros medios generalistas (radio) o especializados, además del mayor e internet, móvil o redes sociales.

Resultan también muy interesantes los estudios que recaban la opinión del mayor o su presencia social y económica como 'nuevos consumidores', a partir de las teorías que consideran hitos sociodemográficos concretos y su impacto de gran calado en la sociedad, como el fenómeno del baby-boom (Taylor, 1995; Hudson, 2009). Con el estudio multimedia relacionado con la representación del mayor, comprendemos además que los medios no son sólo importantes en sí mismos para el mayor, sino que además determinan en gran medida en la elección de otras actividades, con efectos importantes en este sentido (Robinson, 1972, 1981) y la percepción de la edad y la realidad social (Davis, 1984; Gerbner et al., 1980).

El reto consiste en continuar el estudio del mayor desde su realidad compleja, así como, para la industria de la comunicación y de la publicidad, asumir el papel cambiante y protagonista del mayor en nuestras sociedades, y por ende, en los medios de comunicación y la publicidad.

\section{Bibliografía}

\subsection{Libros:}

NIELSEN, A.C. (2000). Report on TV. New York: A.C. Nielsen Company. Nueva York.

KRISTENSEN, B., et al. (2007): Comunicación e persoas maiores. Actas do Foro

Internacional. Colexio Profesional de Xornalistas de Galicia. Santiago de Compostela.

RAMOS-SOLER, I. (2007) El estilo de vida de los mayores y la publicidad. Fundación "La Caixa". Barcelona. 
RODRÍGUEZ, R., \& MORA, K. (2002). Frankenstein y el cirujano plástico: una guía multimedia de semiótica de la publicidad. Universidad de Alicante. Alicante.

STEPHENSON, W. (1953). The study of behavior: Q-technique and its methodology. Chicago: University of Chicago Press. Chicago.

6.2 Artículos:

APARICIO, J. (1988). Las personas mayores en los medios de comunicación de masas. Revista Española de Geriatría y Gerontología, 113-116.

ARONOFF, C. (1974). Old Age in Prime-time. Journal of Communication (24), 86-87.

ATKINS, T.V., JENKINS, M.C., \& PERKINS, M.H. (1991). Portrayal of persons in TV commercials age 50 and older. Psychology: A Journal of Human Behavior, 28, 30-37.

BALAZS, A. L. (1995). The use and image of mature adults in health care advertising (1954-1989). Health marketing Quaterly, XII (3), 13-26.

BELL, J. (1992). In search of a discourse on Aging: The elderly on Television. The Gerontological Society of America , XXXII (3), 305-311.

BERMAN, L., \& NELSON, J. Voltaire's portrayal of old age. Int'l J. Aging and Human Development, Vol. 24(3).

BING ZHANG, Y., \& HARWOOD, J. (2004). Modernization and tradition in an age of globalization: Cultural values in Chinese Television Commercials. Journal of Communication, 54, 156-172.

BING ZHANG, Y., HARWOOD, J., WILLIAMS, A., YLÄNNE-MCEWEN, V., WADLEIGH, P. M., \& THIMM, C. (2006). The Portrayal of Older Adults in Advertising: A Cross-National Review. Journal of Language and Social Psycho$\log y, 264-282$.

BRINGUÉ, X., \& DE LOS ÁNGELES, J. (2000). La investigación académica sobre publicidad, televisión y niños: antecedentes y estado de la cuestión. Comunicación y Sociedad, Vol. XIII, 1, 37-70.

CARRETÓN, M. C., \& RAMOS-SOLER, I. (2010). Redes sociales para mayores y/o mayores en las redes sociales. Icono $14,15-24$.

CARRIGAN, M., \& SZMIGIN, I. (1998). The usage and portrayal of older models in contemporary consumer advertising. Journal of Marketing Practice: Applied Marketing Science , IV (8), 231-248.

CARRIGAN, M., \& SZMIGIN, I. (1999). The portrayal of older characters in magazine advertising. Journal of Marketing Practice: Applied Marketing Science, V (6/7/8), 248-261.

CARRIGAN, M., \& SZMIGIN, I. (1999). The representation of older people in advertisements. Journal of the Market Research Society, XLI (3), 311-326.

CARRIGAN, M., \& SZMIGIN, I. (2000). Does advertising in the UK need older models? The Journal of Product \& Brand Management, IX (2), 128-143.

CARRIGAN, M., \& SZMIGIN, I. (2003). Regulating ageism in UK advertising an industry perspective. Marketing Intelligence and Planing , XXI (4), 198-204. 
CASSATA, M.; ANDERSONA, P. \& SKILL, T. (1980). The older adult in daytime serial drama. Journal of Communication, 30, 48-49.

CHENG, H. (1998). Toward an understanding of cultural values manifest in advertising: A content analysis of Chinese Television Commercials in 1990 and 1995. Journalism and Mass Communication Quaterly, 74, 4, 773-796

CHENG, H., \& SCHWEITZER, J.C. (1996). Cultural values reflected in Chinese and U.S television commercials. Journal of advertising research. Vol 36(3), May-Jun 1996, 27-45.

DAVIS, B., \& FRENCH, W. A. (1989). Exploring advertising usage segments among the aged. Journal of Advertising Research, XXIX (1), 22-29.

DUBOIS, L. (1997). La représentation du viellissement à la télévision: Des images de négation et l'exclusion dans une logique de mise en marché. Canadian Journal on Aging , 354-372.

ELLIOT, J.A. (1984). The daytime television drama portrayal of older adults. The gerontologist, 24, 628-633.

ENGLAND, P., KUHN, A., \& GARDNER, T. (1981). The ages of men and women in magazine advertisements. Journalism Quaterly, 58, 468-471.

FERNÁNDEZ-CID, M., MARTÍN, A., \& CÁCERES M.D. (2008). La imagen de las personas mayores en los medios de comunicación de masas y la opinión pública. UDP- Obra Social Caja Madrid (Colección de estudios)

FESTERVAND, T. A., \& LUMPKIN, J. R. (1984). Response of Elderly Consumers to their portrayal by advertisers. Current Issues \& Research in Advertising

FREIXAS, A. (1998). "La mires como la mires, no la verás". El doble estándar del envejecimiento en la publicidad televisiva. Comunicación y Cultura (3), 29-40.

GANTZ, W., GATENBERG, H. M., \& RAINBOW, C. K. (1980). Approaching Invisibility: The Portrayal of the Elderly in Magazine Advertisements. Journal of Communication, 56-60.

GERBNER, G., GROSS, L., SIGNORELLI, N., \& MORGAN, M. (1980). Aging with television: Images on Television Drama and Conceptions of Social Reality. Journal of Communication.

GONZÁlEZ, M. (1979). Television and People's Images of Old Age. [No Publicada]. Tesis de la Universidad de Pennsylvania

GOODMAN, R. I. (1990). Television News Viewing by Older Adults. Journalism Quarterly, LXVII (1), 137-141.

GRECO, A. J. (1988). Representation of the elderly in advertising: crisis or inconsequence? The Journal of Services Marketing , II (3), 27-34.

GRECO, A. J. (1988). The elderly as communicators: Perceptions of advertising practitioners. Journal of Advertisement Research , 39-46.

HARWOOD, J., ANDERSON, K. (2002) The presence and Portrayal of Social Groups on Prime-time Television. Communication Reports, Vol. 15, 2.

HEALEY, T., \& ROSS, K. (2002). Growing old invisibly: older viewers talk television. Media, Culture and Society, XXIV, 105-120. 
HIEMSTRA, R., GOODMAN, M., MIDDLEMISS, M. A., VOSCO, R., \& ZIEGLER, N. (1983). How older persons are portrayed in television advertising: implications for educators. Educational Gerontology, 111-123.

HODGETTS, D., CHAMBERLAIN, K., \& BASSETT, G. (2003). Between television and the audience: Negotiating representations of ageing. Health: An interdisciplinary journal for the social study of health, illness and medicine, 417-438.

HUDSON, S. (2009). Wooing zoomers: marketing to the mature traveler. Marketing Intelligence and Planing, 444-461.

KESSLER, E.-M., RAKOCZY, K., \& STAUDINGER, U. M. (2004). The portrayal of older people in prime-time television series: the match with gerontological evidence. Ageing and Society, 531-552.

KVASNICKA, B., BEYMER, B., \& PERLOFF, R. (1982). Portrayals of the elderly in magazine advertisements. Journalism Quarterly , 4 (59), 656-658.

LEE, B., KIM, B.-C., \& HAN, S. (2006). The portrayal of older people in television advertisements: a cross-cultural content analysis of the United States and South Korea. Int'l J. Aging and Human Development, LXIII (4), 279-297.

MCCONATHA, J.T., SCHNELL, F., \& MCKENNA, A. (1999). Description of older adults as depicted in magazine advertisements. Psychological Reports, 1999, 85, 1051-1056.

MILLER, D.W., LEYELL, T., \& MAZACHEK, J. (2004). Stereotypes of the elderly in US Television commercials from de 1950s to 1990s. Int'l. J. Aging and human development, Vol 58(4), 315-340.

MULLEY, G. (2007). Myths of Ageing. Clinical Medicine, VII (1), 68-72.

NORTHCOTT, H.C. (1975). Too young, too old- Age in the world of TV. The Gerontologist, 15, 184-186

PETERSON, R. T. (1992). The depiction of senior citizens in magazine advertisements: a content analysis. Journal of Business Ethics (11), 701-706.

PETERSON, R. T. (1995). The portrayal of senior citizens by banks in newspaper advertisements: A content analysis. Journal of Professional Services Marketing, XII (2), 95-106.

POCHINTESTA, P. (2012). De cuerpos envejecidos: un estudio de caso desde el discurso publicitario. Pensar la Publicidad, vol. 6, 1, 163-181.

PRIELER, M., KOHLBACHER, F., HAGIWARA, S., \& ARIMA, A. (2008). The Representation of Older People in Japanese Television Advertising. German Institute for Japanese Studies. Tokio.

PRIELER, M., KOHLBACHER, F., HAGIWARA, S., \& ARIMA, A. (2011). Gender representation of older people in Japanese television advertisements. Sex Roles .

RAMAN, P., HARWOOD, J., WEIS, D., ANDERSON, J.L.,\& MILLER, G. (2008). Portrayals of older adults in US and Indian magazine advertisement: A cross-cultural comparison. The Howard Hournal of Communications, 19: 221-240.

RAMOS-SOLER, I., \& CARRETÓN-BALLESTER, M. (2012). Presencia y representación de las personas mayores en publicidad televisiva. Revista Española de Geriatría y Gerontología , 55-61. 
RAMOS-SOLER, I., \& MANCEBO-ARACIL, J.F. (2013). La investigación sobre personas mayores y publicidad: análisis metodológico (1976-2012). Estudios sobre el Mensaje Periodístico. Vol. 19 Núm. Especial abril, 945-952

ROBERTS, S. D., \& ZHOU, N. (1997). The 50 and older characters in the advertisements of Modern Maturity: Growing older, getting better? Journal of Applied Gerontology, 208-220.

ROBINSON, J.; SKILL, T. \& TURNER, J. (2004). Media usage patterns and portrayal of seniors. Handbook of communication and aging research. 423-446.

ROBINSON, T., \& UMPHREY, D. (2006). First-and third-person perceptions of images of older people in advertising: an inter-generational evaluation. Int'l $J$. Aging and Human Development, 159-173.

ROBINSON, T., \& UMPHREY, D. (2007). Negative Stereotypes Underlying other-person of the Elderly. Educational Gerontology, 309-326.

ROBINSON, T., GUSTAFSON, R., \& POPOVICH, M. (2008). Perceptions of Negative stereotypes of older people in magazine advertisements: comparing the perceptions of older adults and college students. Ageing and Society, 233-251.

ROBINSON, T., POPOVICH, M., GUSTAFSON, R., \& FRASER, C. (2003). Older adults' perceptions of offensive senior stereotypes in magazine advertisements: results of a method analysis. Educational Gerontology (29), 503-519.

ROY, A., \& HARWOOD, J. (1997). Underrepresented, positively portrayed: older adults in television commercials. Journal of Applied Communication Research (25), 39-56.

SCHEWE, C. D. (2001). Effective communication with our Aging Population. Business Horizons, 19-25.

SCHEWE, C. D., \& BALAZS, A. L. (1990). Playing the part: Older adults play many different roles. American Demographics, 24-30.

SCHMITT, K., DUYCK WOOLF, K., \& ANDERSON, D.R. (2003). Viewing the viewers: Viewing behaviors by children and adults during television programs and commercials. Journal of Communication, june 2003.

SHINAR, D. (1982). The portrayal of the elderly in four Israeli daily newspapers. Journalism Quarterly, vol 59 n1 p92-96,111.

SIGNORELLI, N. (1983). Health, prevention and TV: Images of the elderly and perceptions of Social reality. Prevention in Human Services: Aging and prevention, 3, 97-117.

SIGNORELLI, N. (2004). Aging on TV: Messages relating to gender, race and occupation in prime-time. Journal of Broadcasting \& Electronic Media 48, 279-301.

SIGNORELLI, N., \& BACUE, A. (1999). Recognition and respect: A content analysis of prime-time television characters across three decades. Sex Roles, 40, 527-544.

SIMCOCK, P., \& SUDBURY, L. (2006). The invisible majority? Older models in UK television advertising. International Journal of Advertising , I (25), 87-106.

SMITH, M. C. (1976). Portrayal of the Elderly in Prescription Drug Advertising. The Gerontogist , 16 (4), 329-334. 
SMITH, R. B., MOSCHIS, G. B., \& MOORE, R. L. (1987). Social Effects of advertising and personal communication on the elderly consumer. Advances in Marketing and Public Policy, 65-92.

SMITH, R. B., MOSCHIS, G. P., \& MOORE, R. L. (1987). Some advertisements effects on the ederly consumer. Social Effects of Advertising and personal communication (1), 149-152.

SPRING, J. (1993). Seven days of play. American Demogrphics, 15, 50-55.

STEPHENS, N., \& WARRENS, R. A. (1983). Advertising Frequency Requeriments for Older Adults. Journal of Advertisement Research , 23-32.

SWAYNE, L. E., \& GRECO, A. J. (1987). The portrayal of older Americans in television commercials. Journal of Advertising, XVI (1), 47-54.

SZMIGIN, I., \& CARRIGAN, M. (2001). Learning to love the older consumer. Journal of Consumer Behaviour , I (1), 22-34.

TAYLOR, S (1995). Primetime's big sleep. Modern Marturity, 38-44.

THOMPSON, N. J., \& THOMPSON, K. E. (2007). Can marketing practice keep up with Europe's ageing population? Marketing (41).

TUPPER, M. (1995). The representation of elderly persons in prime-time television advertising. School of Mass Communications.

URSIC, A., URSIC, M., \& URSIC, V. (2006). A longitudinal study of the uses of the elderly in magazine advertising. Journal of Consumer Research, (13), 131-133.

VALLS-FERNÁNDEZ, F., \& MARTÍNEZ-VICENTE, J. M. (2007). Gender stereotypes in Spanish television commercials. Sex Roles, 691-699.

VASIL, L. \& WASS, H. (1993) Portrayal of the elderly in the media: A literature review and implications for educational gerontologists. Educational Gerontology, 19, 71-85.

WESTERHOF, G. J., HARINK, K., VAN SELM, M., STRICK, M., \& VAN BAAREN, R. (2010). Filling a missing link: the influence of portrayals of older characters in television commercials on the memory perfomance of older adults. Ageing and Society (30), 897-912.

YE, Y., \& WARD, K.E. (2010). The depiction of illness and related matters in two top-ranked primetime network medical dramas in the United States: A content analysis. Journal of Health Communication, 15: 555-570.

ZHOU, N., \& CHEN, M. Y. (1992). Marginal Life after 49: A preliminary study or the Portrayal of Older People in Canadian Consumer Magazine Advertising. International Journal of Advertising, 343-354.

\section{El autor}

Licenciado en Publicidad y RRPP por la Universidad de Alicante (2004). Máster en Internacionalización por UMH (2005). CAP, Universidad de Alicante (2005). Diploma de Estudios Avanzados (DEA) por la Universidad de Alicante (2009). 